\title{
Aged Rats Are Impaired on an Attentional Set-Shifting Task Sensitive to Medial Frontal Cortex Damage in Young Rats
}

\author{
Morgan D. Barense, Matthew T. Fox, and Mark G. Baxter ${ }^{1}$ \\ Department of Psychology, Harvard University, Cambridge, Massachusetts 02138, USA
}

\begin{abstract}
Normal aging is associated with disruption of neural systems that subserve different aspects of cognitive function, particularly in the hippocampus and frontal cortex. Abnormalities in hippocampal function have been well investigated in rodent models of aging, but studies of frontal cortex function in aged rodents are few. We tested young (4-5 mo old) and aged (27-28 mo old) male Long-Evans rats on an attentional set-shifting task modified slightly from previous publication. After training on two problems in which the reward was consistently associated with the same stimulus dimension, and a reversal of one problem, a new problem was presented in which the reward was consistently associated with the previously irrelevant stimulus dimension (extradimensional shift [EDS]). Aged rats as a group were significantly impaired on the EDS, although some individual aged rats performed as well as young rats on this phase. In addition, some aged rats were impaired on the reversal, although a group effect did not reach significance in this phase. Impairment in neither reversal nor EDS was associated with impairments in spatial learning in the Morris water maze. Young rats with neurotoxic lesions of medial frontal cortex are also selectively impaired on the EDS. These results indicate that normal aging in rats is associated with impaired medial frontal cortex function. Furthermore, age-related declines in frontal cortex function are independent of those in hippocampal function. These results provide a possible basis for correlating age-related changes in neurobiological markers in frontal cortex with cognitive decline.
\end{abstract}

Cognitive aging in humans and nonhuman primates is marked by an impairment in cognitive functions that require the frontal lobe. For example, aged nonhuman primates are severely impaired in a spatial-delayed response task (Bartus et al. 1978; Rapp and Amaral 1989) that is dependent on dorsolateral prefrontal cortex function (Goldman and Rosvold 1970; Bachevalier and Mishkin 1986). In addition, aged humans and nonhuman primates display difficulties in modifying behavior appropriately in response to changing environmental contingencies, a phenomenon called perseveration (Albert and Stafford 1986; Voytko 1993; Lai et al. 1995). Perseveration is characteristic of damage to orbital frontal areas (Jones and Mishkin 1972; Bechara et al. 1998). Despite this evidence of age-related frontal lobe dysfunction in humans and nonhuman primates, little attention has been given to the effects of aging on the functional integrity of frontal lobe regions in the rat (Gallagher and Rapp 1997).

The studies that have examined the integrity of the frontal cortex in the aged rat provide substantial evidence for age-related frontal dysfunction, but the exact locus of the dysfunction is unclear. Winocur and Moscovitch (1990)

${ }^{1}$ Corresponding author.

E-MAIL mbaxter@wjh.harvard.edu; FAX 617-496-2988.

Article and publication are at http://www.learnmem.org/cgi/doi/ $10.1101 / \mathrm{lm} .48602$ and Winocur (1992a,b) compared the performance of young rats with hippocampal or frontal lesions with the performance of aged rats on tasks of complex maze learning, conditional discrimination, and delayed match-tosample. The impairments shown by the aged rats indicated both frontal lobe and hippocampal dysfunction. Zyzak et al. (1995) tested aged rats on an odor-guided recognition memory task, which depends on the integrity of the orbital prefrontal and perirhinal-entorhinal cortex (Otto and Eichenbaum 1992), and the Morris water maze, which depends on the hippocampus (Morris et al. 1982; Gallagher and Holland 1992) but not the medial prefrontal cortex (de Bruin et al. 1994). These investigators observed considerable variation in the performance of aged rats on the acquisition of the odor-recognition memory task; however, there was no association between impaired acquisition of this task and spatial-learning ability in the water maze. Because selective orbital prefrontal cortex lesions in young rats produce selective deficits on olfactory but not spatial learning (Eichenbaum et al. 1983), and severely impair acquisition of the odor-recognition memory task (Otto and Eichenbaum 1992), selective dysfunction of the orbital prefrontal area could account for the specific deficit in odor-recognition memory unrelated to deficits in spatial performance.

Information about region-specific impairments in aged rats is needed because the exact region of dysfunction in

LEARNING \& MEMORY 9:191-201 @ 2002 by Cold Spring Harbor Laboratory Press ISSN1072-0502/02 \$5.00

$$
\begin{array}{llllllllllllllll} 
& E & A & R & N & I & N & G & \mathbf{Q} & M & E & M & O & R & Y \\
\text { www.learnmem.org } & & &
\end{array}
$$


the frontal cortex of aged rats remains unclear. Attentional set-shifting tasks provide a possible approach to localizing the area of frontal dysfunction in the aged rat. Performance on tests of executive function in humans has long been believed to be dependent on intact frontal lobe function (for review, see Moscovitch and Ulmita 1991). However, the observed impairments were not always specific to selective frontal lobe dysfunction: Generalized or diffuse brain damage (i.e., Robinson et al. 1980) or damage to specific nonfrontal regions could produce similar deficits (i.e., Canavan et al. 1989; Corkin 2001). Attentional-shifting tasks require that the subject inhibit previously acquired responses that are inappropriate to the present situation. There are two types of shifts: intradimensional shifts (IDSs) and extradimensional shifts (EDSs). In an IDS discrimination, the subject must apply a discrimination rule to a new problem, which is based on the same perceptual dimension (e.g., color) that was attended to in the previous problem. In contrast, an EDS requires that the subject attend to a different perceptual dimension from that attended to in the previous problem to solve the new problem.

Owen et al. (1991) tested a group of adult human patients with localized excisions of the frontal lobes on such an attentional set-shifting task and compared their performance with adult and elderly control subjects without brain damage. Subjects with frontal lobe damage were found to be significantly impaired relative to young controls in their ability to perform the EDS but not in their ability to attend to novel exemplars of the previously relevant dimension (IDS). Moreover, there were no qualitative or quantitative differences between the performance of the frontal lobe group and the group of elderly controls, indicating that the pattern of deficits in the elderly group stemmed from frontal lobe dysfunction. In the same study, patients with unilateral temporal lobe lesions and patients who had undergone surgical amygdalo-hippocampal removal were not impaired on the EDS. A subsequent study in a larger group of healthy human subjects also reported age-related declines in EDS performance (Robbins et al. 1998). In a neuroimaging study, Rogers et al. (2000) scanned human subjects learning IDS, EDS, and reversal visual-discrimination problems. Relative to IDS learning, EDS learning differentially activated left polar and right dorsolateral regions of the prefrontal cortex. In contrast, reversal learning activated the left caudate nucleus.

The nonhuman primate prefrontal cortex has also been shown to mediate shifts in attention between perceptual dimensions of complex stimuli. Dias, Robbins, and Roberts (1996a, 1997) found that damage to the lateral prefrontal cortex in marmoset monkeys selectively impaired performance on the EDS, whereas damage to the orbitofrontal cortex selectively impaired reversal performance. Neither lesion affected performance on the IDS. A recent preliminary report of a similar deficit in macaque monkeys with dorsolateral prefrontal damage (Moore et al. 2001) strongly indicates that EDS specifically requires intact dorsolateral prefrontal function, a conclusion supported by human neuroimaging studies (Rogers et al. 2000). An additional preliminary report of impairments in attentional set shifting in aged monkeys (Moore et al. 1998) supports the view that cognitive aging in primates is also associated with impairment in frontal cortex-dependent attentional processes.

Birrell and Brown (2000) have developed an attentional set-shifting task in rats that is formally identical to the task used in monkeys and humans. Bilateral lesions of the medial frontal cortex of the rat result in normal acquisition of attentional set but selectively impair performance on the EDS in this test paradigm. This impairment mirrors that seen in primates with lesions of the lateral prefrontal cortex, leading to the conclusion that the medial frontal cortex of the rat has functional similarity to primate lateral prefrontal cortex. Hence, this task may be particularly well suited to examining the functional integrity of medial frontal cortex in aged rats, an area that bears some functional homology with dorsolateral prefrontal cortex in primates.

The development of a model of aging of frontal lobe function in the rat would be extremely beneficial to the study of cognitive aging. Such a model would permit neurobiological studies of the mechanism of age-related declines in frontal lobe function, as well as provide a system for testing potential therapeutic interventions. The use of rats as subjects confers an enormous advantage over the use of nonhuman primates. Because of the scarcity of aged nonhuman primates, aging studies in nonhuman primates incur an enormous cost of obtaining and maintaining sufficient numbers of subjects to achieve adequate statistical power. The shorter lifespan of rats makes aged subjects more readily available. However, the homology of frontal cortical areas of the rat with those of the primate is somewhat unclear, particularly with regard to whether the rat possesses an analog of primate dorsolateral prefrontal cortex (Preuss 1995). Some details of connectivity differ between rodents and primates (for review, see Granon and Poucet 2000). Despite these differences, there is striking functional similarity between the medial frontal cortex of the rat and the dorsolateral prefrontal cortex of the primate (i.e., Birrell and Brown 2000; for review, see Granon and Poucet 2000; Brown and Bowman 2002). In both species, the prefrontal cortex appears to have a role in aspects of cognitive functioning that are referred to as executive functions; more specifically, the medial frontal cortex in rats and the dorsolateral prefrontal cortex in primates have a crucial role in these processes.

In the present research, we tested aged rats on an attentional set-shifting task adapted from Birrell and Brown (2000) to investigate the effects of aging on medial frontal cortex function. In this task, rats learned a series of discrimination problems that required them to dig in one of two

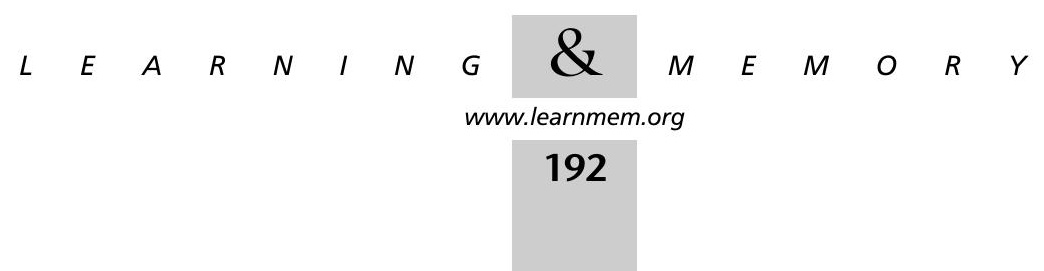




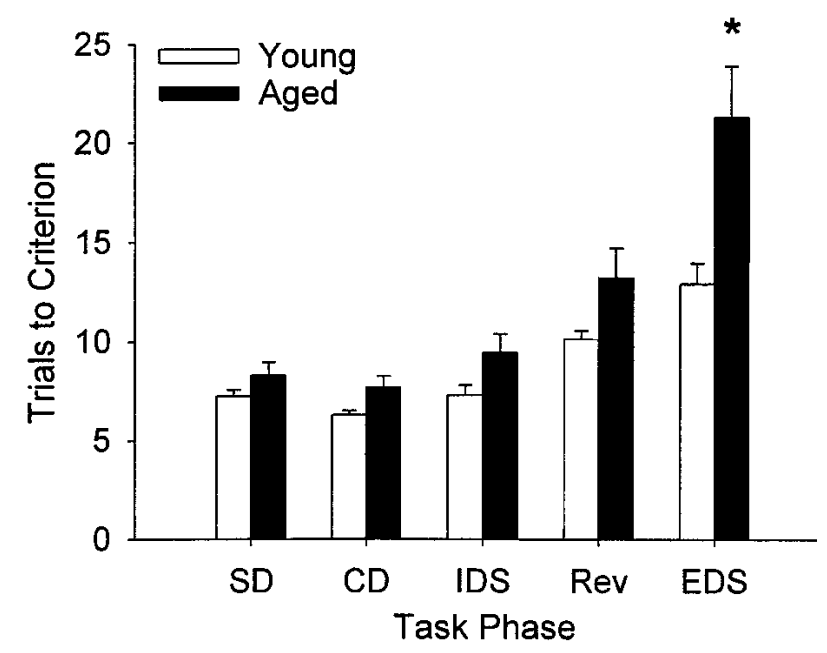

Figure 1 Acquisition of each discrimination problem in aged and young rats. Trials to criterion represent the number of trials required to meet a criterion of six consecutive correct responses, including the criterion run. Aging results in a selective impairment on extradimensional shift (EDS) learning; there was no difference between the performance of young and aged rats on any of the phases, with the exception of the EDS in which aged rats were significantly impaired. Error bars indicate standard error of mean (SEM).

pots to retrieve a cereal reward. One of two perceptual dimensions could be relevant for finding the reward: digging medium or odor (placed on the rim of the pot). Rats learned the task in five phases, two in which the two dimensions were gradually introduced, followed by an IDS, a reversal of the IDS, and an EDS. These same rats were tested in the Morris water maze to examine whether age-related changes in hippocampal function were independent of presumed age-related changes in medial frontal function. The dependent measure of water-maze learning was the learning-index score developed by Gallagher, Burwell, and Burchinal (1993), which is based on probe-trial performances and indexes the accuracy of search for the platform.

\section{RESULTS}

\section{Discrimination Learning}

Figure 1 shows the trials to criterion for each of the discriminations. Aged rats (27-28 mo old) performed comparably to young rats (4-5 mo old) on all phases of discrimination learning except for the EDS in which they were significantly impaired. There was a difference in performance dependent on the particular phase, and the effect of age differed between the phases. Analysis of variance (ANOVA) with age and initial discrimination dimension as between-subject factors and task phase as a within-subject factor revealed a main effect of age $\left(F_{[1,22]}=15.03\right.$, $p=.001)$; main effect of task phase, $\left(F_{[4,88]}=27.03\right.$, $p<.0005)$; and an age by task phase interaction,
$\left(F_{[4,88]}=2.88, p=.027\right)$. These effects did not depend on which dimension (medium or odor) the rats were initially trained: The main effect of initial discrimination dimension was not significant $\left(F_{[1,22]}=1.32, p=.26\right)$, nor were interactions of this effect with the other effects in the analysis ( $p$ s $>$.20). Post hoc analyses (Bonferroni-corrected $t$ tests) revealed that the effect of age on task performance was specific to the EDS $(t[24]=2.98,5 p=.035)$; age-related differences in other task phases were not significant $(5 p>.175)$.

Nevertheless, aged rats on average performed numerically worse on all task phases, raising the concern that a subtle overall impairment in discrimination learning was only revealed on the more challenging EDS. This possibility was refuted by calculating correlations between EDS performance and performance on other phases of the task in the aged rats; these correlations ranged from -0.271 to $-0.024, p \mathrm{~s}>.37$, except the EDS-reversal correlation in aged rats $(r=.529, p=.06)$. Thus, EDS performance in aged rats is independent of performance on all other phases, with the exception of the reversal. The EDS-reversal relationship is shown in Figure 2. Some aged rats perform as well as young rats on both EDS and reversal. It is interesting that among the aged rats that are impaired on EDS (i.e., scoring outside the range of young rat performance), there

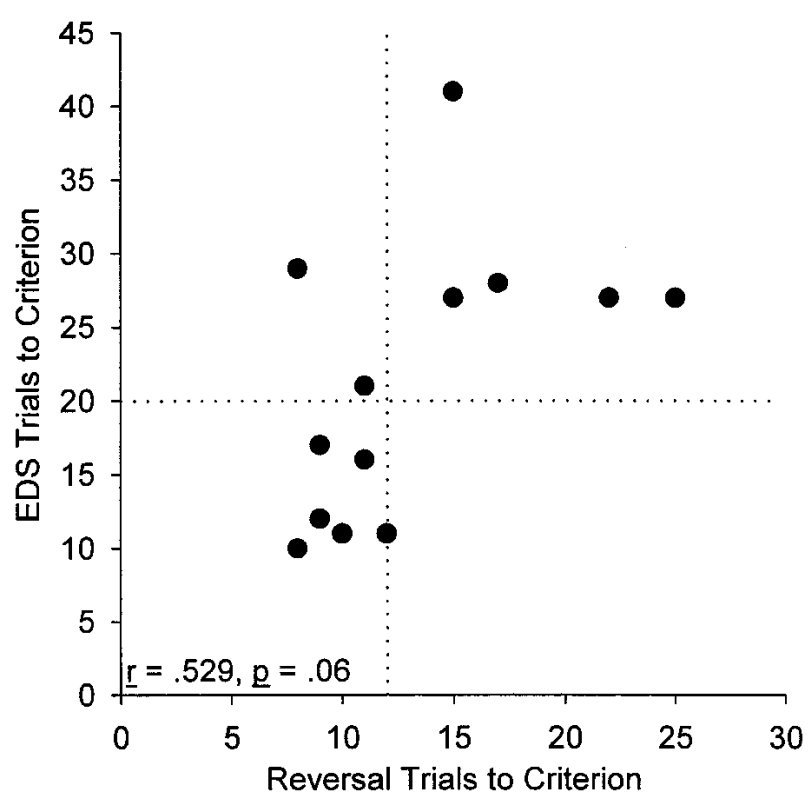

Figure 2 Relationship between EDS and reversal performance in aged rats. Performance on reversal was the only phase of discrimination learning that showed a correlation with performance on EDS. The dotted lines indicate the maximum number of trials to criterion for any subject in the young group on each phase. There are substantial individual differences in EDS performance in aged rats, with some performing as well as young rats on both the EDS and reversal. It is interesting that of the rats that are impaired on the EDS, there does not appear to be an association between EDS and reversal performance.

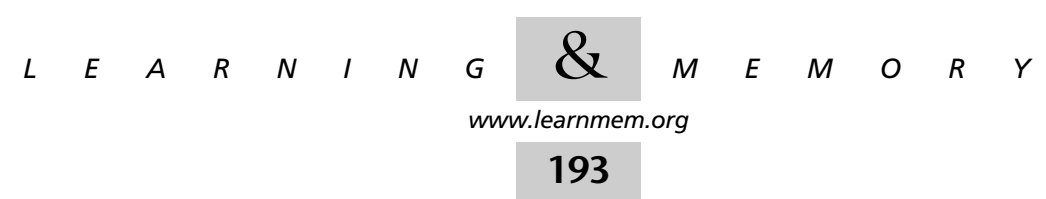


does not appear to be an association between EDS performance and reversal performance. It is also interesting that in our sample, no aged rats were impaired on reversal but unimpaired on EDS. Correlations between task performance in all phases for young and aged rats are given in Table 1.

Within-subject comparisons confirmed that the EDS and reversal were more challenging than the IDS in young rats. Comparisons of the performance of young rats on the EDS and reversal with performance on the IDS revealed that both the reversal and the EDS were more difficult to acquire than the IDS $(t[12]=-4.85, p<.0005, t[12]=-4.78$, $p<.0005$, respectively). This validates the set-shifting task, because the IDS and EDS are both new discrimination problems, but the EDS is more challenging than the IDS.

\section{Morris Water Maze}

Aged rats were impaired on water-maze performance. Comparison of young and aged rats revealed an age-related difference on both learning index $(t[24]=2.37, p=.026)$ and, unexpectedly, performance on cue training as well $(t[23]=3.59, p=.002)$; cue-training data for one aged rat were lost. The mean learning index for the aged rats was 279.6 (standard error of mean $[\mathrm{SEM}]=13.6$ ) and 235.4 for the young rats $(\mathrm{SEM}=12.8)$; a higher learning-index score corresponds to poorer performance. The mean cue-training swim time was $11.3 \mathrm{sec}(\mathrm{SEM}=1.35)$ for the old rats and $5.49 \mathrm{sec}(\mathrm{SEM}=0.91)$ for the young rats.

Impaired cue-training performance raises the possibility that the spatial-learning impairment in the aged rats (re-

\begin{tabular}{|c|c|c|c|c|c|c|c|}
\hline Young & SD & $C D$ & IDS & Rev & EDS & Index & Cue time \\
\hline SD & - & & & & & & \\
\hline CD & 0.293 & - & & & & & \\
\hline IDS & -0.079 & 0.683 & - & & & & \\
\hline $\operatorname{Rev}$ & -0.728 & -0.045 & 0.169 & - & & & \\
\hline EDS & 0.042 & -0.049 & -0.034 & -0.041 & - & & \\
\hline Index & 0.307 & 0.147 & -0.309 & -0.169 & 0.441 & - & \\
\hline Cue time & 0.377 & -0.200 & 0.104 & -0.530 & 0.060 & -0.237 & - \\
\hline Aged & SD & CD & IDS & Rev & EDS & Index & Cue time \\
\hline SD & - & & & & & & \\
\hline CD & 0.220 & - & & & & & \\
\hline IDS & 0.489 & 0.235 & - & & & & \\
\hline $\operatorname{Rev}$ & -0.155 & 0.597 & -0.089 & - & & & \\
\hline EDS & -0.271 & -0.024 & -0.221 & 0.529 & - & & \\
\hline Index & 0.164 & -0.211 & 0.199 & 0.004 & 0.192 & - & \\
\hline Cue time & -0.356 & -0.289 & 0.070 & -0.474 & -0.442 & -0.337 & - \\
\hline
\end{tabular}

$\mathrm{SD}$, simple discrimination; CD, compound discrimination; IDS, intradimensional shift; Rev, reversal; EDS, extradimensional shift; Index, spatial-learning index; Cue time, swim time on cue-training trials (average of six trials). Correlations with cue-training swim time in aged rats are based on 12 observations instead of 13 , because data for one aged rat were lost. Correlations of absolute value $>0.553$ ( 0.576 for cue-training swim time in the aged rats) are significant at the .05 level, uncorrected for multiple comparisons. flected in the learning-index score) is caused by some sensorimotor or motivational deficit. However, there was no correlation between learning index and cue-training swim time $(r=-.337$ for the aged rats and $r=-.237$ for young rats). This lack of correlation indicates that the learningindex scores are likely to be accurate reflectors of hippocampal-dependent spatial-learning ability rather than some other noncognitive deficit.

\section{Relationship Between Attentional Set Shifting and Spatial Learning}

All correlations between water-maze performance measures and trials to criterion in the different phases of set shifting are given in Table 1. Performance on the discrimination problems was independent of performance on the water maze in aged rats. The learning index was not correlated with trials to criterion on each phase; correlations ranged from -0.211 to 0.199 , $p$ s $>.489$ (Fig. 3). This supports an independence of age-related impairment in spatial learning and attentional set shifting. Similarly, no correlations between set-shifting performance and learning index emerged in the young rats ( $r$ between -.309 and $.441, p s>.13)$. With regard to cue-training performance, a correlation between cue-training swim time and errors to criterion on reversal approached significance in both young and aged rats ( $r=-.53$ and $-.474, p=.063$ and .119 , respectively). This correlation is somewhat curious, because it indicates that better reversal performance (fewer trials to criterion) was associated with poorer cue-training performance (longer time to reach the platform). No other correlations between set shifting and cue-training latency approached significance in either age group $(r$ between -.442 and $.377, p s>.151)$.

The cue-training impairment in our aged rats, although mild, raises concerns because it is not usually seen in aged rats of this strain (Gallagher et al. 1993), and because an impairment in cue training after place learning is associated with medial prefrontal lesions (de Bruin et al. 1994). Hence, our population of aged rats may be unusual in some way, and the impairments in cue learning and EDS performance could be linked. By this view, impaired medial prefrontal function in our aged sample might be idiosyncratic. However, it is worth noting that performance on cue training and EDS are, if anything, negatively correlated (Table 1), that is,

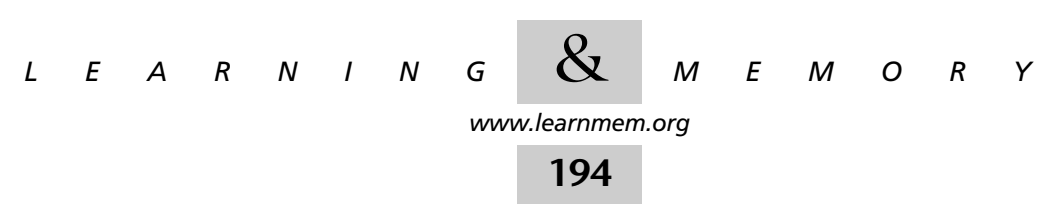



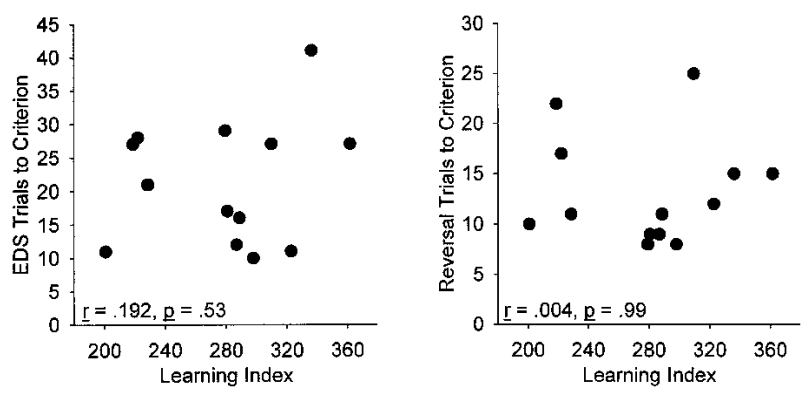

Figure 3 Relationship between water-maze performance and set shifting in aged rats. Examination of the relationship between performance of aged rats on the water maze and $\operatorname{EDS}(A)$ and on the water maze and reversal $(B)$ reveals no correlation between spatial learning and either phase of performance of the attentional setshifting task.

poorer cue training is associated with better EDS performance, rather than the converse, which would be expected if the impairments were related.

\section{DISCUSSION}

In the present experiment, we compared the performance of young and aged rats on two different tasks, attentional set shifting and the Morris water maze. We found that aged rats as a group were significantly impaired in their ability to shift their attentional set to a previously irrelevant dimension (EDS) but not in any other phase of the attentional setshifting task. Because young rats with neurotoxic lesions of the medial frontal cortex are also selectively impaired on the EDS (Birrell and Brown 2000), our results indicate that normal aging in rats is associated with impaired medial frontal cortex function. Some aged rats showed an impairment in learning the reversal, although there was not a significant group effect.

Although aged rats were impaired in spatial learning in the water maze, consistent with the findings of many other studies (Gage et al. 1984; Gallagher and Burwell 1989; Gallagher et al. 1993), their spatial-learning ability was unrelated to performance on the attentional set-shifting discrimination problems, indicating that the age-related decline in medial frontal cortex function is unrelated to impaired hippocampal function in normal aging. Together, these results indicate that during normal cognitive aging of rats, different regions decline independently of each other. The present study provides a useful behavioral model for selectively probing neurobiological changes in prefrontal function associated with aging.

\section{Performance on EDS as a Neuropsychological Probe for Medial Frontal Function in the Rat}

Damage to the lateral or dorsolateral prefrontal cortex results in a selective deficit in EDS performance in humans (Owen et al. 1991), marmoset monkeys (Dias et al. 1996a,b, 1997), and macaque monkeys (Moore et al. 2001). Human neuroimaging studies also find that performance on the EDS activates the dorsolateral prefrontal cortex (Rogers et al. 2000). In the rat, medial frontal cortex lesions selectively impair EDS performance (Birrell and Brown 2000). The present study adds to these findings by providing evidence of impaired prefrontal function in aged rats that extends to a selective deficit in EDS performance.

We used performance on EDS as an indicator of medial frontal function in aged rats on the basis of the effects of medial frontal lesions in young rats in the similar experimental paradigm of Birrell and Brown (2000). Our current findings closely parallel their results; aged rats performed comparably well to young rats on all phases of the discrimination learning except for the EDS, in which they were significantly impaired, similar to young rats with medial frontal cortex lesions. Thus, this task provides a useful behavioral model for probing neurobiological changes in medial frontal function associated with aging in the rat.

There is the concern that the effect observed is not specific to the medial frontal cortex and that any cortical damage will cause deficits in EDS performance. Given the numerous studies providing evidence for the selectivity of the EDS deficit, this seems unlikely. Nevertheless, impairments in medial temporal lobe function in aging, including hippocampal dysfunction, are well documented (Gallagher et al. 1995; Gallagher and Rapp 1997), raising the possibility that such dysfunction could account for the deficit in EDS performance seen in the present study. However, we found no correlation between performance on EDS and on the water maze, indicating that the neural substrates underlying these impairments are independent of each other. Given that water-maze performance depends on the integrity of the hippocampus (Morris et al. 1982; Gallagher and Holland 1992), the lack of correlation between the two tasks confirms that the EDS deficit cannot be the result of hippocampal decline.

In one study, entorhinal lesions resulted in equal rates of acquisition of the IDS and the EDS, whereas controls acquired the IDS more rapidly (Oswald et al. 2001), indicating a role for the entorhinal cortex in attentional function. Thus, the possibility that the EDS deficit observed in the present study is the result of entorhinal cortex dysfunction should be considered. However, the attentional set-shifting task used by Oswald et al. (2001) is very different from the present task in that it required the rat to combine novel exemplars of the formerly irrelevant dimension with the previously reinforced stimuli of a relevant dimension and ignore novel stimuli of the other previously reinforced dimension. Hence, findings from this task may not generalize to our testing procedure, in which novel exemplars were used for both IDS and EDS. In any case, Zyzak et al. (1995) found no evidence of perirhinal-entorhinal cortical dysfunction in their aged rats (which were of the same strain and age as those tested in the present study) on the basis of

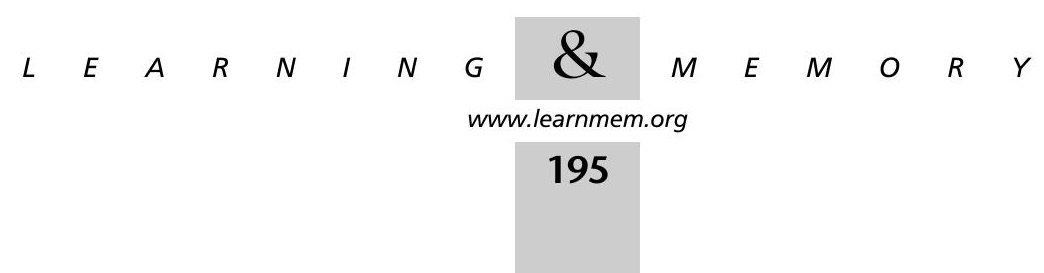


normal performance across delays in the olfactory nonmatching-to-sample task. Hence, it is also unlikely that perirhinal-entorhinal cortex dysfunction accounts for the reversal impairments observed here.

In a recent study, we found that damage to the posterior parietal cortex resulted in a selective EDS deficit (M.T. Fox et al., in prep.), thus creating the concern that the impairment observed in the present study was not the result of medial frontal cortex dysfunction. Hence, the impairment in our aged rats could reflect dysfunction of medial prefrontal cortex, posterior parietal cortex, or a disconnection between the two structures. This question could be addressed by testing aged rats on a cognitive task sensitive to parietal damage, but not frontal damage, and examining the relationship of impairment on that task (if any) with performance on EDS. It is worth noting that other data indicate that attentional functions of the posterior parietal cortex are intact in normal aging. For example, Baxter and Voytko (1996) found that spatial orienting of attention as measured by a cued reaction task was unimpaired in aged monkeys. This same task is impaired by damage to the parietal cortex in humans (Posner et al. 1984). The lack of impairment of aged monkeys in this task indicates that this neural system seems to be functionally intact in aging, and thus parietal cortex dysfunction is less likely to account for the EDS impairment seen in the present study.

A final possibility is that age-related impairment in striatal function (e.g., Burwell et al. 1995; Nicolle et al. 1996) could contribute to impaired set shifting. For example, patients with Parkinson's disease, who have compromised basal ganglia function, are impaired in attentional set-shifting tasks (Downes et al. 1989; Owen et al. 1993), and inactivation of the striatum in rats impairs strategy switching in a discrimination-learning task (Ragozzino et al. 2002). Although the effects of striatal lesions on this task have not specifically been determined, marmoset monkeys with dopamine depletion in the caudate nucleus are not impaired on attentional set shifting when the EDS is initially administered and only show an impairment when they are required to reengage attention to previously irrelevant dimensions (Collins et al. 2000; Crofts et al. 2001). Hence, because disruption of striatal function by dopamine depletion produces a different pattern of deficit in the set-shifting task, we tentatively conclude that age-related impairments in striatal function also cannot account for our results.

\section{Performance on Reversal}

Although as a group rats were not impaired in learning the reversal, some individual rats showed an impairment at this phase. Previous studies that have investigated the effects of aging on reversal in nonhuman primates have found marked impairments of aged animals in reversing stimulus-reward associations. For example, impairments in object reversal (Bartus et al. 1979; Peters et al. 1996; Moss et al. 1999;
Voytko 1999) and spatial-reversal learning (Lai et al. 1995; Peters et al. 1996; Moss et al. 1999) have been observed, although intact spatial (Voytko 1999) and object-reversal learning (Rapp 1990; Lai et al. 1995) have also been reported. The inconsistencies between these studies can most likely be explained by the fact that impairments on reversal may only be apparent the first time a reversal is encountered (for review, see Baxter 2001). Schoenbaum et al. (2002) tested aged rats on an odor-discrimination reversal task and found that aged rats as a group were moderately impaired in acquiring the reversal-discrimination problems. Moreover, although aged rats as a group were impaired on the water maze, there was no association between reversal and water-maze performance.

Lesion studies have helped to localize the neural substrate underlying reversal deficits to the orbital frontal cortex (Jones and Mishkin 1972; Eichenbaum et al. 1983; Meunier et al. 1997). Rats with lesions of orbital frontal cortex are impaired on the reversal but not the EDS phase of the set-shifting task (Brown and Bowman 2002), similar to the effect of orbital frontal lesions in nonhuman primates (Dias et al. 1996a,b, 1997). The individual differences observed in aged rats in our study are consistent with the individual differences observed in other studies that investigated reversal impairments with aging in nonhuman primates and rats (Lai et al. 1995; Voytko 1997, 1999; Schoenbaum et al. 2002).

It is possible that with a larger sample size, a group effect on reversal learning in aged rats would have been statistically significant. It is also possible that because the reversal was learned more rapidly than the EDS, it may not be as sensitive a measure to selective orbital frontal dysfunction as EDS performance is to medial frontal dysfunction. It is interesting to note that reversal performance was the only phase of learning that correlated with performance on the EDS (Fig. 2). When divided into quadrants on the basis of the maximum number of errors made by young rats, an interesting relationship emerges. Some rats performed equally well as young rats on both the EDS and the reversal, indicated by the subjects in the bottom left quadrant of Figure 2. However, among the aged rats impaired on the EDS (upper two quadrants), there does not appear to be a correlation between EDS performance and reversal performance. It is also worth noting that in our sample, every aged rat that showed an impairment on the reversal was also impaired on the EDS, although there were some subjects that showed an impairment on EDS but not on reversal.

Despite the absence of a significant group effect on reversal learning, we tentatively conclude that the reversal impairment observed in individual aged rats in the present study is a result of age-related orbital frontal cortex dysfunction. Medial frontal dysfunction probably cannot account for reversal impairment; although there was a correlation between EDS and reversal performance in the aged rats, this

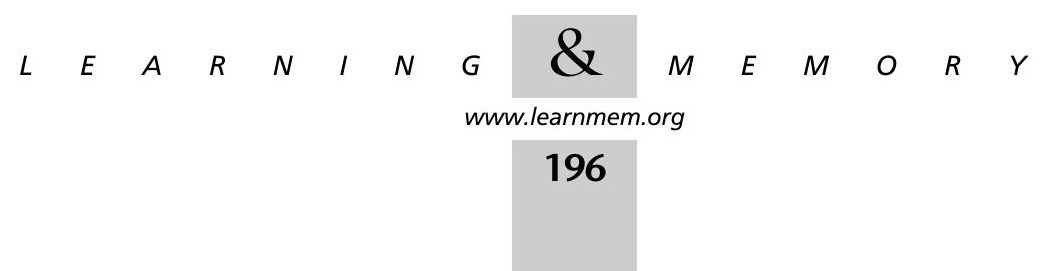


appeared to stem from an absence of aged rats that were impaired in reversal but not EDS. Furthermore, medial frontal cortex lesions are without effect on reversal performance in young rats in this task (Birrell and Brown 2000). Dysfunction of other brain regions, namely hippocampus and rhinal cortex, could also produce a reversal impairment. Although hippocampal damage can cause impairments on reversal learning (Mahut 1971; Becker et al. 1981; Murray et al. 1998), there was no correlation between performance on the water maze and performance on the reversal in this study, indicating that hippocampal damage cannot account for the observed reversal impairments. Damage to the perirhinal-entorhinal (rhinal) cortex is also associated with impairments in reversal learning (Murray et al. 1998), although again, given that Zyzak et al. (1995) found no evidence of perirhinal-entorhinal cortical dysfunction in their aged rats, damage to the perirhinal-entorhinal cortex is unlikely to account for the reversal impairments observed here.

The lack of a group effect of aging on reversal learning and the fact that every rat that was impaired on reversal was also impaired on EDS indicates that orbital frontal cortex may be more resistant to age-related decline than the medial frontal cortex. This might tentatively indicate that the progression of dysfunction seems to be that decline in the medial frontal cortex occurs before decline in the orbital frontal cortex. This speculation could be addressed directly by a cross-sectional study in which rats of different ages are tested in this task.

\section{Toward a Model of Aging in the Prefrontal Cortex of the Rat}

The utility of a model of cognitive aging in the rat prefrontal cortex depends on the extent of homology between the prefrontal cortex of the rat and that of the primate. If rats do not have a prefrontal cortex, then the study of its role in cognition is limited to nonhuman primates. As mentioned in the beginning of the article, the existence of a cortical area in the rat homologous with primate dorsolateral prefrontal cortex is controversial (Preuss 1995; Öngür and Price 2000). Nevertheless, at least with regard to the aspects of executive function engaged by the attentional set-shifting task, the medial frontal cortex of the rat seems to represent a functional homolog to primate lateral prefrontal cortex (Birrell and Brown 2000; Brown and Bowman 2002).

Cognitive aging does not appear to consist of a single process that progressively impairs the functions of different neural systems in parallel; rather, different neural systems decline independently in aging, with substantial differences across individuals (for review, see Gallagher and Rapp 1997; Baxter 2001). This phenomenon was seen in the present study in which declines in prefrontal cortex function in aged rats (indexed by the reversal and EDS phases of the task) were uncorrelated with performance in the Morris water maze, an index of hippocampal function. The attentional set-shifting task appears to provide a reasonable basis for evaluating prefrontal function in aged rats. Because performance on this task appears to be independent of hippocampal function, impaired spatial-information processing in aged rats should not contaminate performance. Furthermore, administration of the task is very rapid and requires only a few days to complete, a serious consideration when dealing with very old animals, when compared with other operant tasks that may require months to achieve stable performance.

Relative to age-related alterations in hippocampal function in aging rats, comparatively little is known about agerelated changes in prefrontal cortex function, and virtually nothing is known about the functional significance of agerelated changes in prefrontal function in terms of their impact on cognitive functions that specifically require prefrontal cortex. A candidate mechanism for disruption of prefrontal function in aging is loss of excitatory amino acid (glutamate) receptors, which decline in prefrontal cortex of aged rats and mice (Le Jeune et al. 1996; Magnusson 1998). It is also quite possible that neurobiological changes that disrupt the function of the aged prefrontal cortex are different from those that disrupt the aged hippocampus. Indeed, in normal young animals, there is evidence that activation of intracellular signaling cascades (such as protein kinase A) can have opposite effects on memory in prefrontal cortex as in hippocampus (Taylor et al. 1999). Investigation of neurobiological markers in prefrontal cortex of aged rats tested on prefrontal-dependent cognitive tasks like the attentional set-shifting procedure will provide insight into the biological mechanisms of cognitive aging in this brain region.

\section{MATERIALS AND METHODS}

\section{Discrimination Learning}

\section{Subjects}

Twenty-six Long Evans male rats (Charles River) were housed individually in $25 \times 46 \times 20$-cm plastic cages. Aged rats were acquired at 3 mo of age and pair-housed until 14-16 mo of age, at which time they were housed individually. Testing was conducted during the light phase of a 12-h light/dark cycle, beginning at $8 \mathrm{a} . \mathrm{m}$. The rats were maintained on a restricted diet with the amount of food administered contingent on their performance on the foodmotivated task. A weight of $85 \%$ of ad lib body weight served as a guideline; certain individual aged rat subjects were briefly reduced below this weight to motivate performance because their baseline weights were relatively high (670-835 g). Water was freely available in the home cage. At the time of discrimination testing, aged rats were 27-28 mo old and young rats were 4-5 mo old.

\section{Apparatus}

Rats can readily be trained to dig in small bowls filled with sawdust to retrieve food reward (e.g., Wood et al. 1999). The experiment used terra-cotta flower pots as digging bowls, with an internal di-

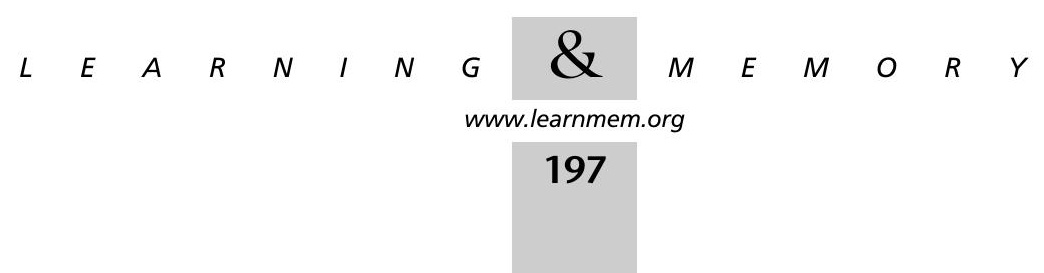


ameter and depth of $10 \mathrm{~cm}$. The food reward was a half of a Honey Nut Cheerio (General Mills). The rim of the pot could be scented with perfumed oils (Body Shop perfume oils or Florasense Oils), and the terra-cotta absorbed the scent to produce a long-lasting odor.

The test apparatus was a Plexiglas box measuring $50 \times 37.5 \times$ $25 \mathrm{~cm}$ with an opaque Plexiglas barrier separating one third of the box from the rest. The two digging pots were placed adjacent to each other in the larger section of the box while the rat waited in the smaller section. The rat was given access to the pots by raising the divider, which was then lowered once the trial had begun.

\section{Habituation}

Testing procedures were adapted from Birrell and Brown (2000). On the day before testing, rats were placed in the testing box and given access to two pots filled with the bedding used in their home cages and baited with several Cheerios. The cups were rebaited continuously until the rats were digging reliably to retrieve the food rewards. This session lasted $\sim 30 \mathrm{~min}$. At the end of this period, rats were trained sequentially on two simple discrimination (SD) problems. One consisted of odor of rose versus sensual in home-cage bedding, and the other consisted of medium of crumpled tissue paper versus shredded latex gloves with no added odor. Each SD was trained to a criterion of six consecutive correct trials. The order of the two SDs and positive stimulus were randomized across rats, with the stimulus set for each aged rat pair-matched to one young control. These stimuli were not used again in subsequent testing.

\section{Testing Paradigm}

A trial began by raising the barrier to allow the rat access to the two digging pots, only one of which was baited. The first four trials of each discrimination constituted a discovery period in which the rat was allowed to dig in both pots. An error was recorded if the rat first dug in the unbaited pot. After the first four trials were completed, the rat was only allowed to dig in one of the two pots. If the rat dug first in the unbaited pot, it was returned to the smaller section of the box and not permitted to find the food reward in the other pot. Testing continued until the rat reached a criterion of six consecutive correct trials on a discrimination problem. The next discrimination in the series was introduced immediately after criterion was reached on the preceding problem. Occasionally a rat would stop searching for rewards in the middle of the test session during the beginning trials of the EDS (two aged rats); in these cases the session was ended and continued on the next day.

In a single test session, rats performed a series of discriminations paralleling the procedure used by Birrell and Brown (2000) (Table 2). Initially, an SD between either two odors or two digging media was presented, followed by a compound discrimination (CD) with the same positive stimulus as the initial SD. If the SD was an odor discrimination, the cups were filled with a neutral medium (corncob bedding). If the SD was a medium discrimination, the media were presented in two unscented cups. In the $\mathrm{CD}$, the new dimension that was introduced did not reliably predict the location of the food reward. An IDS was then presented; the IDS was another compound discrimination in which both the particular stimuli were changed, but the relevant dimension (either odor or medium) remained the same. The IDS problem was then reversed, so that the formerly negative stimulus became the positive stimulus (and vice versa), with the irrelevant dimension still not predictive of the location of the reward. Finally, the rats were presented with an EDS in which the formerly irrelevant dimension became the
Table 2. Example of a Possible Combination of Stimulus Pairs for a Rat Shifting from Digging Medium to Odor as the Relevant Dimension

\begin{tabular}{|c|c|c|c|c|}
\hline \multirow[b]{2}{*}{ Discrimination } & \multicolumn{2}{|c|}{ Dimension } & \multicolumn{2}{|c|}{ Exemplar combinations } \\
\hline & Relevant & Irrelevant & $\mathrm{S}+$ & S- \\
\hline SD & Medium & & M1 & M2 \\
\hline \multirow[t]{2}{*}{$\mathrm{CD}$} & Medium & Odor & M1/O1 & $\mathrm{M} 2 / \mathrm{O} 2$ \\
\hline & & & M1/O2 & M2/O1 \\
\hline \multirow[t]{2}{*}{ IDS } & Medium & Odor & M3/O3 & $\mathrm{M} 4 / \mathrm{O} 4$ \\
\hline & & & M3/O4 & M4/O3 \\
\hline \multirow[t]{2}{*}{ Reversal } & Medium & Odor & M4/O3 & $\mathrm{M} 3 / \mathrm{O} 4$ \\
\hline & & & M4/O4 & M3/O3 \\
\hline \multirow[t]{2}{*}{ EDS } & Odor & Medium & O5/M5 & O6/M6 \\
\hline & & & O5/M6 & O6/M5 \\
\hline
\end{tabular}

Half of the rats switched from medium to odor and half switched from odor to medium. The correct examplar is shown in bold and can be paired with either exemplar from the irrelevant dimension. In the IDS and EDS, the stimuli were novel exemplars of each dimension.

relevant one, and the previously relevant dimension no longer held predictive value.

Eight of the rats in each group were trained with medium as the relevant dimension in the $\mathrm{SD}, \mathrm{CD}$, IDS, and reversal, and shifted to odor for the relevant dimension of the EDS; the other five rats in each group were trained in the opposite direction, with odor as the relevant dimension for the first four phases. (The death of several aged rats after the counterbalancing was designed caused the unequal division of rats between medium and odor as the initial training dimension.) The particular stimuli used for each discrimination problem (Table 3) were also randomized across subjects. All media included a small amount of powdered Cheerios reward to discourage the rat from trying to sniff out the hidden reward. For example, a rat shifting from odor to medium might first encounter an SD between mulberry and patchouli in cups filled with neutral (corncob) bedding. The reward would consistently be located in the cup with one of the pair of scents, for example, patchouli. On reaching criterion on this discrimination, the $\mathrm{CD}$ would be introduced with the same two odors but now with two different media. For instance, the rat could encounter a mulberry cup filled with foam

Table 3. Stimulus Pairs Used

\begin{tabular}{ll}
\hline Odor pairs & \multicolumn{1}{c}{ Medium pairs } \\
\hline Jasmine vs. vanilla & Foam rubber vs. plastic beads \\
Mulberry vs. patchouli & $\begin{array}{l}\text { Gravel vs. BBs } \\
\text { Pine shavings vs. shredded } \\
\text { manila folders }\end{array}$ \\
\hline
\end{tabular}

The exemplars within a dimension were always used in pairs, for example, whenever jasmine appeared as one odor within a discrimination, the other odor was vanilla. No two rats within the same group received the same combinations, but the aged and control groups were matched. The order of presentation of exemplars and the combination of exemplars into positive (+) and negative (-) stimuli was determined by a pseudorandom series generated before testing.

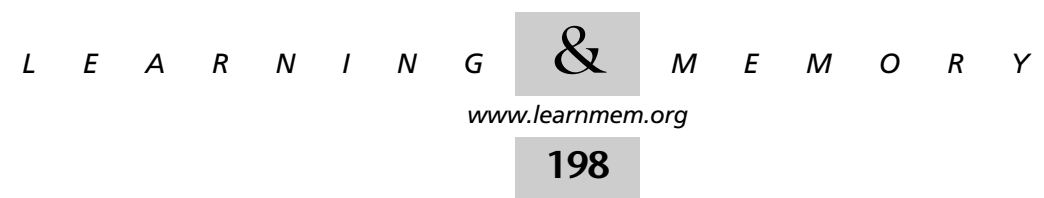


rubber and a patchouli cup with plastic beads or a mulberry cup with plastic beads and a patchouli cup with foam rubber. In either case, the reward would be located in the patchouli cup regardless of the medium filling the cup. On reaching criterion on the $\mathrm{CD}$, the IDS would be introduced with an entirely new set of exemplars. For example, this rat would be presented with a jasmine cup filled with gravel and a vanilla cup filled with BBs or a jasmine cup filled with BBs and a vanilla cup filled with gravel. Again, regardless of the medium, the food reward was consistently associated with one of the scents, for example, vanilla. Once the rat reached criterion on the IDS, the reversal would be introduced. On these trials, exactly the same stimuli would be presented as in the IDS, but now the jasmine cup always contained the food reward. On reaching criterion on the reversal, the EDS would be introduced with the third set of entirely new exemplars. For example, the rat would be presented with a cinnamon cup filled with pine shavings and a mulberry cup filled with shredded manila folders or a cinnamon cup filled with shredded manila folders and a mulberry cup filled with pine shavings. Now the reward would be consistently associated with one of the media (e.g., pine shavings) regardless of the odor scenting the cup.

There were too many possible pairings and orderings of stimuli to permit full counterbalancing; therefore each stimulus was matched with a pair (e.g., when jasmine was the positive stimulus, vanilla was always the negative stimulus). The order of presentation of stimuli was counterbalanced to the greatest degree possible across rats. Particular care was taken to ensure that each exemplar was the positive stimulus of the EDS for at least one pair of subjects and the negative stimulus for another. With the exception of pair-matched controls, no two subjects were presented the stimuli in the same order.

\section{Morris Water Maze}

Four to $6 \mathrm{wk}$ before testing on the discrimination tasks, the young and aged groups had been tested in the Morris water maze according to standard procedures (e.g., Baxter et al. 1995). A water maze (diameter $180 \mathrm{~cm}$ ) was filled with an opaque mixture of white, water-soluble Crayola paint and water that was heated to $27^{\circ} \mathrm{C}$ before the administration of experimental trials. A collapsible Plexiglas platform $(12 \mathrm{~cm} \times 12 \mathrm{~cm}$ surface $)$ extended from the floor of the tank to $\sim 1.5 \mathrm{~cm}$ below the surface of the water. The tank was surrounded by black curtains and illuminated from above by four 60-watt lights. Four high-contrast, black and white visual cues (4 $\mathrm{ft} \times 3 \mathrm{ft}$ ) were affixed to the curtains, positioned at the North, East, South, and West sides of the tank, respectively. A white sheet covered the ceiling; a small video camera for tracking the rats' movements protruded through the center of the sheet. An HVS VP-200 tracking system and VCR were connected to the video camera, and each rat's data were collected using HVS Water for Windows (HVS Image).

For the initial place-learning problem, the platform was placed in the Southeast quadrant. Three trials per day were given each day for $8 \mathrm{~d}$. On each trial, the rat was placed in the pool along the perimeter of the tank facing the tank wall. The four entry points, North, East, South, and West, were randomized across trials, but the same sequence of start points was used for each rat. Standard learning trials ended when the rat located the escape platform, or when $90 \mathrm{sec}$ had elapsed, at which time the rat was guided to the platform. In either case, the rat was allowed to remain on the platform for $15 \mathrm{sec}$ before being returned to a holding cage for a 30-sec intertrial interval. Every sixth trial (i.e., the last trial on days
$2,4,6$, and 8 ) was a probe trial during which the platform was lowered to the bottom of the pool for the first $30 \mathrm{sec}$ of the trial, making it unavailable for escape, and was then raised using a concealed drawstring. The rat was then allowed an additional $60 \mathrm{sec}$ to locate and escape onto the platform. On the day after the completion of the place-learning problem, six cue-training trials were given. The collapsible platform was replaced with a moveable circular platform, $10 \mathrm{~cm}$ in diameter, with black tape around the edge. The surface of the platform was 1-2 $\mathrm{cm}$ above the surface of the water in the pool. The platform was moved to different positions in the maze on each trial and placed in the center of one of the four quadrants according to a predetermined pseudorandom order. The rat was allowed $30 \mathrm{sec}$ to reach the platform on each trial. The intertrial interval for cue-training trials was $15 \mathrm{sec}$. The time taken by the rat to swim to the platform on each trial was recorded.

The primary measure of performance in the water maze was a learning index calculated from performance on probe trials. This index was calculated as described by Gallagher et al. (1993) and was a weighted sum of average proximity to the platform on the second, third, and fourth probe trials. The formula used was $1.26 \times$ (second probe proximity) $+1.43 \times$ (third probe proximity) +1.43 $\times$ (fourth probe proximity). Because the water-maze protocol was identical to that used by Gallagher et al. (1993) and the same strain of rats were used, we thought it appropriate to use the same multipliers to derive the learning-index scores. Our average learningindex scores are somewhat higher than those reported by Gallagher et al. (1993), indicating that the physical characteristics of our maze environment (e.g., positioning of cues and lighting) may make acquisition of the place problem somewhat more challenging in our laboratory. We also examined average swim time on cuetraining trials to determine whether sensorimotor impairments in the aged rats might have influenced performance in the spatial phase of the water-maze task.

\section{ACKNOWLEDGMENTS}

We are very grateful to Dr. Verity Brown for her advice in establishing the set-shifting task in our laboratory and Drs. Michelle Nicolle and Joshua Rodefer for helpful comments on the manuscript. We also thank Dr. Joanne Berger-Sweeney for her contributions to this research. This work was supported by NIA Grant R03-AG17337. Mark G. Baxter is an Alfred P. Sloan Research Fellow.

The publication costs of this article were defrayed in part by payment of page charges. This article must therefore be hereby marked "advertisement" in accordance with 18 USC section 1734 solely to indicate this fact.

\section{REFERENCES}

Albert, M.S. and Stafford, J.L. 1986. CT scan and neuropsychological relationships in aging and dementia. In Advances in clinical neuropsychology (eds. G. Goldstein and R.E. Taske), Vol. 3, pp. 31-53. Plenum, New York.

Bachevalier, J. and Mishkin, M. 1986. Visual recognition impairment follows ventromedial but not dorsolateral prefrontal lesions in monkeys. Behav. Brain Res. 20: 249-261.

Bartus, R.T., Fleming, D., and Johnson, H.R. 1978. Aging in the rhesus monkey: Debilitating effects on short-term memory. J. Gerontol. 33: 858-871.

Bartus, R.T., Dean, III, R.L., and Fleming, D.L. 1979. Aging in the rhesus monkey: Effects on visual discrimination learning and reversal learning. J. Gerontol. 34: 209-219.

Baxter, M.G. 2001. Cognitive aging in nonhuman primates. In Functional neurobiology of aging (eds. P.R. Hof and C.V. Mobbs), pp. 407-419. Academic Press, San Diego, CA.

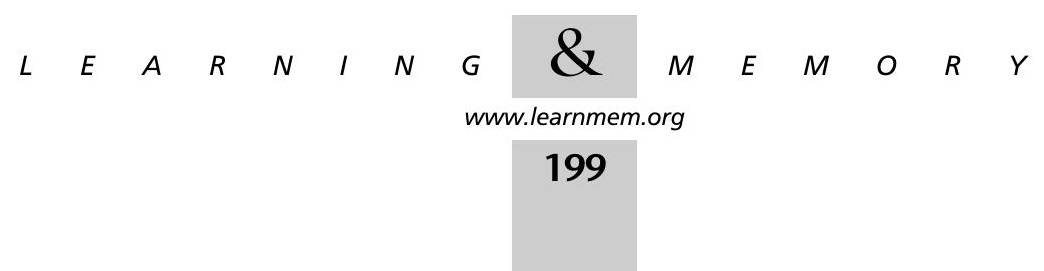


Baxter, M.G. and Voytko, M.L. 1996. Spatial orienting of attention in adult and aged rhesus monkeys. Behav. Neurosci. 110: 898-904.

Baxter, M.G., Bucci, D.J., Gorman, L.K., Wiley, R., and Gallagher, M. 1995. Selective immunotoxic lesions of basal forebrain cholinergic cells: Effects on learning and memory in rats. Behav. Neurosci. 109: 714-722.

Bechara, A., Damasio, H., Tranel, D., and Anderson, S.W. 1998. Dissociation of working memory from decision making within the human prefrontal cortex. J. Neurosci. 18: 428-437.

Becker, J.T., Olton, D.S., Anderson, C.A, and Breitinger, E.R.P. 1981. Cognitive mapping in rats: The role of the hippocampal and frontal systems in retention and reversal. Behav. Brain Res. 3: 1-22.

Birrell, J. and Brown, V. 2000. Medial frontal cortex mediates perceptual attentional set shifting in the rat. J. Neurosci. 20: 4320-4324.

Brown, V.J. and Bowman, E.M. 2002. Rodent models of prefrontal cortical function. Trends Neurosci. 25: 340-343.

Burwell, R.D., Lawler, C.P., and Gallagher, M. 1995. Mesostriatal dopamine markers in aged Long-Evans rats with sensorimotor impairment. Neurobiol. Aging 16: 175-186.

Canavan, A.G.M., Passingham, R.E., Marsden, C.D., Quinn, N., Wyke, M., and Polkey, C.E. 1989. The performance on learning tasks of patients in the early stages of Parkinson's disease. Neuropsychologia 27: 141-156.

Collins, P., Wilkinson, L.S., Everitt, B. J., Robbins, T.W., and Roberts, A.C. 2000. The effect of dopamine depletion from the caudate nucleus of the common marmoset (Callithrix jacchus) on tests of prefrontal cognitive function. Behav. Neurosci. 114: 3-17.

Corkin, S. 2001. Beware of frontal lobe deficits in hippocampal clothing. Trends Cognit. Sci. 5: 321-323.

Crofts, H.S., Dalley, J.W., Collins, P., Van Denderen, J.C., Everitt, B.J., Robbins, T.W., and Roberts, A.C. 2001. Differential effects of 6-OHDA lesions of the frontal cortex and caudate nucleus on the ability to acquire an attentional set. Cereb. Cortex 11: 1015-1026.

de Bruin, J.P.C., Sánchez-Santed, F., Heinsbroek, R.P.W., Donker, A., and Postmes, P. 1994. A behavioural analysis of rats with damage to the medial prefrontal cortex using the Morris water maze: Evidence for behavioural flexibility, but not for impaired spatial navigation. Brain Res. 652: 323-333.

Dias, R., Robbins, T.W., and Roberts, A.C. 1996a. Dissociation in prefrontal cortex of affective and attentional shifts. Nature 380: 69-72.

1996b. Primate analogue of the Wisconsin card sorting test: Effects of excitotoxic lesions of the prefrontal cortex in the marmoset. Behav. Neurosci. 110: 872-886.

- 1997. Dissociable forms of inhibitory control within prefrontal cortex with an analog of the Wisconsin card sort test: Restriction to novel situations and independence from "on-line" processing. $J$. Neurosci. 17: 9285-9297.

Downes, J.J., Roberts, A.C., Sahakian, B.J., Evenden, J.L., Morris, R.G., and Robbins, T.W. 1989. Impaired extra-dimensional shift performance in medicated and unmedicated Parkinson's disease: Evidence for a specific attentional dysfunction. Neuropsychologia 27: 1329-1343

Eichenbaum, H., Clegg, R.A., and Feelely, A. 1983. Reexamination of functional subdivisions of the rodent prefrontal cortex. Exp. Neurol. 79: 434-451.

Gage, F.H., Dunnett, S.B., and Björklund, A. 1984. Spatial learning and motor deficits in aged rats. Neurobiol. Aging 5:43-48.

Gallagher, M. and Burwell, R.D. 1989. Relationship of age-related decline across several behavioral domains. Neurobiol. Aging 10: 691-708.

Gallagher, M. and Holland, P.C. 1992. Preserved configural learning and spatial learning impairment in rats with hippocampal damage. Hippocampus 2: 81-88.

Gallagher, M. and Rapp, P.R. 1997. The use of animal models to study the effects of aging on cognition. Annu. Rev. Psychol. 48: 339-370.

Gallagher, M., Burwell, R., and Burchinal, M. 1993. Severity of spatial learning impairment in aging: Development of a learning index for performance in the Morris water maze. Behav. Neurosci. 107: 618-628
Gallagher, M., Nagahara, A.H., and Burwell, R.D. 1995. Cognition and hippocampal systems in aging: Animal models. In Brain and memory: Modulation and mediation of neuroplasticity (eds. J.L. McGaugh, N. Weinberger, and G. Lynch), pp. 103-126. Oxford University Press, New York.

Goldman, P.S. and Rosvold, H.E. 1970. Localization of function within the dorsolateral prefrontal cortex of the rhesus monkey. Exp. Neurol. 27: 291-304.

Granon, S. and Poucet, B. 2000. Involvement of the rat prefrontal cortex in cognitive functions: A central role for the prelimbic area. Psychobiology 28: 229-237.

Jones, B. and Mishkin, M. 1972. Limbic lesions and the problem of stimulus-reinforcement associations. Exp. Neurol. 36: 362-377.

Lai, Z.C., Moss, M.B., Killiany, R.J., Rosene, D.L., and Herndon, J.G. 1995 Executive system dysfunction in the aged monkey: Spatial and object reversal learning. Neurobiol. Aging 16: 947-954.

Le Jeune, H., Cécyre, D., Rowe, W., Meaney, M.J., and Quirion, R. 1996. Ionotropic glutamate receptor subtypes in the aged memory-impaired and unimpaired Long-Evans rat. Neuroscience 74: 349-363.

Magnusson, K.R. 1998. Aging of glutamate receptors: Correlations between binding and spatial memory performance in mice. Mech Ageing Dev. 104: 227-248.

Mahut, H. 1971. Spatial and object reversal learning in monkeys with partial temporal lobe ablations. Neuropsychologia 9: 409-424.

Meunier, M., Bachevalier, J., and Mishkin, M. 1997. Effects of orbital frontal and anterior cingulate lesions on object and spatial memory in rhesus monkeys. Neuropsychologia 35: 999-1015.

Moore, T.L., Killiany, R.J., Rosene, D.L., and Moss, M.B. 1998. Executive system dysfunction in the aged rhesus monkey using an analog of the Wisconsin card sorting test. Soc. Neurosci. Abstr. 24: 1764.

. 2001. Lesions of dorsal prefrontal cortex produce an executive function deficit in the rhesus monkey. Soc. Neurosci. Abstr. 27: Program no. 533.6.

Morris, R.G., Garrud, P., Rawlins, J.N., and O'Keefe, J. 1982. Place navigation impaired in rats with hippocampal lesions. Nature 297: 681-683.

Moscovitch, M. and Ulmita, C. 1991. Conscious and nonconscious aspects of memory: A neuropsychological framework of modules and central systems. In Perspectives on cognitive neuroscience (eds. R.G. Lister and H.J. Weingartner), pp. 229-266. Oxford University Press, New York.

Moss, M.B., Killiany, R.J., and Herndon, J.G. 1999. Age-related cognitive decline in the rhesus monkey. In Cerebral cortex: Neurodegenerative and age-related changes in structure and function of cerebral cortex (eds. A. Peters and J.H. Morrison), Vol. 14, pp. 21-47. Kluwer Academic/Plenum Publishers, New York.

Murray, E.A., Baxter, M.G., and Gaffan, D. 1998. Monkeys with rhinal cortex damage or neurotoxic hippocampal lesions are impaired on spatial scene learning and object reversals. Behav. Neurosci. 112: 1291-1303.

Nicolle, M.M., Bizon, J., and Gallagher, M. 1996. In vitro autoradiography of ionotropic glutamate receptors in hippocampus and striatum of aged Long-Evans rats: Relationship to spatial learning. Neuroscience 74: 741-756.

Öngür, D. and Price, J.L. 2000. The organization of networks within the orbital and medial prefrontal cortex of rats, monkeys and humans. Cereb. Cortex 10: 206-219.

Oswald, C.J., Yee, B.K., Rawlins, J.N., Bannerman, D.B., Good, M., and Honey, R.C. 2001. Involvement of the entorhinal cortex in a process of attentional modulation: Evidence from a novel variant of an IDS/EDS procedure. Behav. Neurosci. 115: 841-849.

Otto, T. and Eichenbaum, H. 1992. Complementary roles of the orbital prefrontal cortex and the perirhinal-entorhinal cortices in an odor-guided delayed-nonmatching-to-sample task. Behav. Neurosci. 106: $763-776$

Owen, A.M., Roberts, A.C., Polkey, C.E., Sahakian, B.J., and Robbins, T.W. 1991. Extra-dimensional versus intra-dimensional set shifting 
performance following frontal lobe excisions, temporal lobe excisions or amygdalo-hippocampectomy in man. Neuropsychologia 29: 993-1006.

Owen, A.M., Roberts, A.C., Hodges, J.R., Summers, B.A., Polkey, C.E., and Robbins, T.W. 1993. Contrasting mechanisms of impaired attentional set-shifting in patients with frontal lobe damage or Parkinson's disease. Brain 116: 1159-1175.

Peters, A., Rosene, D.L., Moss, M.B., Kemper, T.L., Abraham, C.R., Tigges, J., and Albert, M.S. 1996. Neurobiological bases of age-related cognitive decline in the rhesus monkey. J. Neuropathol. Exp. Neurol. 55: 861-874.

Posner, M.I., Walker, J.A., Friedrich, F.J., and Rafal, R.D. 1984. Effects of parietal injury on covert orienting of attention. J. Neurosci. 4: $1863-1874$

Preuss, T.M. 1995. Do rats have prefrontal cortex? The Rose-Woolsey-Akert program reconsidered. J. Cogn. Neurosci. 7: 1-24.

Ragozzino, M.E., Ragozzino, K.E., Mizumori, S.J.Y., and Kesner, R.P. 2002. Role of the dorsomedial striatum in behavioral flexibility for response and visual cue discrimination learning. Behav. Neurosci. 116: $105-115$.

Rapp, P.R. 1990. Visual discrimination and reversal learning in the aged monkey (Macaca mulatta). Behav. Neurosci. 104: 876-884.

Rapp, P.R. and Amaral, D.G. 1989. Evidence for task-dependent memory dysfunction in the aged monkey. J. Neurosci. 9: 3568-3576.

Robbins, T.W., James, M., Owen, A.M., Sahakian, B.J., Lawrence, A.D., McInnes, L., and Rabbitt, P.M. 1998. A study of performance on tests from the CANTAB battery sensitive to frontal lobe dysfunction in a large sample of normal volunteers: Implications for theories of executive functioning and cognitive aging. Cambridge Neuropsychological Test Automated Battery. J. Int. Neuropsychol. Soc. 4: 474-490.

Robinson, A.L., Heaton, R.K., Lehman, R.A.W., and Stilson, D.W. 1980. The utility of the Wisconsin card sorting test in detecting and localising frontal lobe lesions. J. Consult. Clin. Psychol. 48: 605-614.

Rogers, R.D., Andrews, T.C., Grasby, P.M., Brooks, D.J., and Robbins, T.W.
2000. Contrasting cortical and subcortical activations produced by attentional-set shifting and reversal learning in humans. J. Cogn. Neurosci. 12: 142-162.

Schoenbaum, G., Nugent, S., Saddoris, M.P., and Gallagher, M. 2002. Teaching old rats new tricks: Age-related impairments in olfactory reversal learning. Neurobiol. Aging 23: 555-564.

Taylor, J.R., Birnbaum, S., Ubriani, R., and Arnsten, A.F.T. 1999. Activation of cAMP-dependent protein kinase A in prefrontal cortex impairs working memory performance. J. Neurosci. 19: RC23(1-5).

Voytko, M.L. 1993. Cognitive changes during normal aging in monkeys assessed with an automated test apparatus. Neurobiol. Aging. 14: 643-644.

-1997. Functional and neurobiological similarities of aging in monkeys and humans. Age 20: 29-44.

- 1999. Impairments in acquisition and reversals of two-choice discriminations by aged rhesus monkeys. Neurobiol. Aging 20: 617-627.

Winocur, G. 1992a. A comparison of normal old rats and young adult rats with lesions to the hippocampus or prefrontal cortex on a test of matching-to-sample. Neuropsychologia. 30: 769-781.

- 1992b. Conditional learning in aged rats: Evidence of hippocampal and prefrontal cortex involvement. Neurobiol. Aging 13: 131-135.

Winocur, G. and Moscovitch, M. 1990. Hippocampal and prefrontal cortex contributions to learning and memory: Analysis of lesion and aging effects on maze learning in rats. Behav. Neurosci. 104: 544-551.

Wood, E.R., Dudchenko, P.A., and Eichenbaum, H. 1999. The global record of memory in hippocampal neuronal activity. Nature 397: 613-616.

Zyzak, D.R., Otto, T., Eichenbaum, H., and Gallagher, M. 1995. Cognitive decline associated with normal aging in rats: A neuropsychological approach. Learn. Mem. 2: 1-16.

Received March 14, 2002; accepted in revised form May 21, 2002.

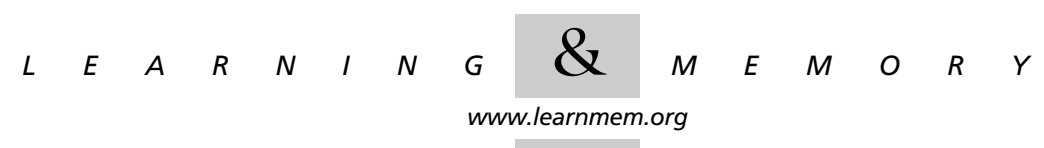




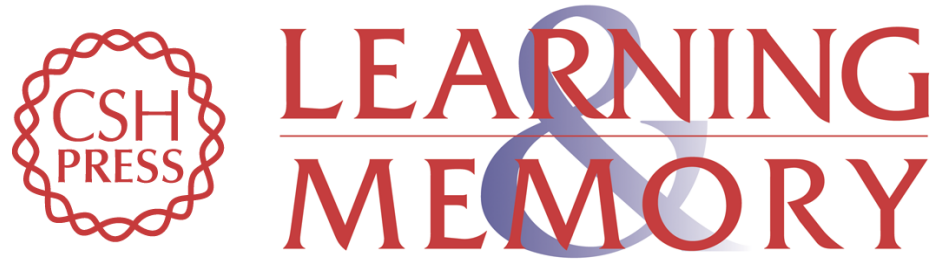

\section{Aged Rats Are Impaired on an Attentional Set-Shifting Task Sensitive to Medial Frontal Cortex Damage in Young Rats}

Morgan D. Barense, Matthew T. Fox and Mark G. Baxter

Learn. Mem. 2002, 9:

Access the most recent version at doi:10.1101/lm.48602

References This article cites 59 articles, 6 of which can be accessed free at: http://learnmem.cshlp.org/content/9/4/191.full.html\#ref-list-1

License

Email Alerting Receive free email alerts when new articles cite this article - sign up in the box at the Service top right corner of the article or click here. 